\title{
The classification of endo-trivial modules
}

\author{
Jon F. Carlson ${ }^{1, \star}$, Jacques Thévenaz ${ }^{2}$ \\ 1 Department of Mathematics, University of Georgia, Athens, Georgia 30602, USA \\ (e-mail: jfc@math.uga.edu) \\ 2 Institut de Géométrie, Algèbre et Topologie, EPFL, CH-1015 Lausanne, Switzerland \\ (e-mail: Jacques. Thevenaz@epfl.ch)
}

Oblatum 2-I-2004 \& 13-V-2004

Published online: 2 September 2004 - (C) Springer-Verlag 2004

\section{Introduction}

This paper provides the final step for the classification of all endo-trivial $k P$-modules, where $P$ is a finite $p$-group and $k$ is a field of characteristic $p$. The classification was obtained in [CaTh2] in the case that every maximal elementary abelian subgroup of $P$ has rank at least 3 . The result had been known for a long time if $P$ has a maximal elementary abelian subgroup of rank 1, i.e. if $P$ is cyclic or generalized quaternion. The purpose of this paper is to handle the only remaining case that $P$ has at least one maximal elementary abelian subgroup of rank 2 . This case is rather subtle and requires special treatment.

Let $T(P)$ be the group of equivalence classes of endo-trivial $k P$-modules. The classification of endo-trivial $k P$-modules is equivalent to the complete description of the structure of $T(P)$. It was proved in [CaTh2] that $T(P)$ is free abelian if $P$ is not cyclic, quaternion or semi-dihedral, and that $T(P) \cong \mathbb{Z}$ in most cases, namely when every maximal elementary abelian subgroup of $P$ has rank at least 3. Assuming now that $P$ has at least one maximal elementary abelian subgroup of rank 2 (but $P$ not semidihedral), we prove that $T(P)$ is free abelian on $r$ explicit generators, which are classes of suitable relative syzygies of the trivial module. The torsionfree rank of $T(P)$, which is $r$, was described in group-theoretic terms by Alperin [Al]. The chief contribution of this paper is to show that the relative syzygies found by Alperin generate all of $T(P)$ and not just a subgroup of finite index. This is the final step in the classification of the elements of $T(P)$.

Our strategy is to solve first the problem for several small $p$-groups, namely all extraspecial and almost extraspecial $p$-groups which satisfy the

\footnotetext{
* Partly supported by a grant from NSF
} 
condition of having a maximal elementary abelian subgroup of rank 2 (they have order at most $p^{5}$ ). Then a group-theoretic argument allows us to deduce the result in general. In the (almost) extraspecial case, we use a method which appears in a different form in [CaTh2] and which turns out to be applicable again with some modification. This consists of decomposing and tensoring endo-trivial modules according to the structure of the variety of a certain quotient of the module. The method was used in [CaTh2] in a proof by contradiction. Here we adapt the method and state positive results which are then used to handle our problem.

As a by-product of the main result, we obtain that $T(P)$ is generated by relative syzygies, except for the case in which $P$ is a generalized quaternion group and an exceptional module is known to exist. This confirms a recent conjecture and is the starting point needed by S. Bouc and N. Mazza in their work [BoMa] on the Dade group of extraspecial $p$-groups. Another consequence of the property that $T(P)$ is generated by relative syzygies is the fact that the classification is independent of the base field $k$ (except for the quaternion group of order 8 , see [CaTh1]). Thus, throughout this paper, $k$ denotes an arbitrary field of characteristic $p$.

We should point out that there is another set of generators for $T(P)$ found by the first author using a different approach [Ca]. These generators are constructed by direct decompositions of the ordinary syzygies of the trivial module using information from group cohomology. The construction uses many of the same methods as this paper, but is completely independent. The generators must coincide with the relative syzygies used in this paper. However, we have no direct proof of the coincidence. Instead, the verification of the isomorphisms of the modules relies on the classification, and in particular, on the fact that $T(P)$ is torsion free [CaTh2]. A third collection of elements, constructed by Bouc and the second author using tensor induction [BoTh], generates a subgroup of $T(P)$ of the correct rank, but which (in general) may not be all of $T(P)$.

The paper is organized roughly as follows. The second and third sections develop some group theoretic information on $p$-groups with maximal elementary abelian subgroups of rank 2 , and describe the restrictions to elementary abelian subgroups of the relative syzygies defined by Alperin. Sections four and five generalize results from [CaTh2] on decompositions and tensor products of endo-trivial modules. In Sect. 6, we prove a classification of the endo-trivial modules for the extraspecial groups of rank 2. The final step in the classification comes in Sect. 7, using restriction to extraspecial subgroups and some group theoretic results.

The first author wishes to thank the Université de Lausanne and the École Polytechnique Fédérale de Lausanne for their kind support and hospitality during several visits while this and other papers in the series were being written. The first author wants also to thank the Alexander von Humboldt Foundation and the Max Planck Institute for Mathematics for their support and hospitality. 


\section{Groups with maximal elementary subgroups of rank 2}

We first recall some known results concerning $p$-groups $P$ having maximal elementary abelian subgroups of rank 2 . Let $\mathcal{A}$ be the poset of all elementary abelian subgroups of $P$ of rank at least 2. Clearly the isolated vertices of $\mathscr{A}$ are precisely the maximal elementary abelian subgroups of rank 2 . Let $\mathcal{B}$ be the subposet of $\mathcal{A}$ consisting of all elementary abelian subgroups of $P$ of rank at least 3 as well as their subgroups of rank 2. The following grouptheoretic result is well-known and not very hard to prove (see Lemma 10.21 in $[\mathrm{GLS}]$ ).

Lemma 2.1. Let $m(P)$ be the maximal rank of an elementary abelian subgroup of $P$.

(a) If $m(P)=2$, then $A$ consists of isolated vertices.

(b) If $m(P) \geq 3$, then $\mathcal{B}$ is a connected component of $\mathcal{A}$ and all other connected components (if any) are isolated vertices.

We shall call $\mathscr{B}$ the big connected component of $\mathcal{A}$.

By a theorem of Alperin [Al], the torsion-free rank of $T(P)$ is equal to the number of conjugacy classes of connected components of $\mathcal{A}$. We shall have to revisit Alperin's theorem in the next section and, to this end, we need the following more precise lemma, which appears in slightly different forms in [Al] and [GLS]. For notation, let $C_{m}$ denote a cyclic group of order $m$, $D_{2 m}$ a dihedral group of order $2 m$, and $Q_{2^{k}}$ a generalized quaternion group of order $2^{k}$.

Lemma 2.2. Let $P$ be a p-group with $m(P) \geq 2$. Let $\mathcal{C}_{1}, \ldots, \mathcal{C}_{r}$ be representatives of the conjugacy classes of connected components of $\mathcal{A}$ and assume that $r \geq 2$. For a suitable choice of numbering, these representatives can be made to satisfy the following conditions:

(a) $\mathcal{C}_{1}$ contains a normal elementary abelian subgroup $E$ of rank 2 , unless $p=2$ and $P$ is dihedral of order $\geq 16$. Moreover, if $m(P) \geq 3$, then $\mathcal{C}_{1}$ is equal to the big component $\mathcal{B}$.

(b) If $2 \leq i \leq r$, then $\mathcal{C}_{i}=\left\{E_{i}\right\}$ where $E_{i}$ is maximal elementary abelian of rank 2. Moreover, $E_{i}$ contains a non-central subgroup $S_{i}$ of order $p$, and we have that $C_{P}\left(S_{i}\right)=S_{i} \times L_{i}$ with $L_{i}$ either cyclic or generalized quaternion.

(c) If $2 \leq i \leq r$, then $C_{P}\left(S_{i}\right)=C_{P}\left(E_{i}\right)$ is a subgroup of index $p$ in $N_{P}\left(E_{i}\right)$ and $N_{P}\left(E_{i}\right)=\left(E \cdot E_{i}\right) * L_{i}$, where $*$ denotes the central product. Moreover $E \cdot E_{i}$ is extraspecial of order $p^{3}$ and exponent $p$ if $p$ is odd, and isomorphic to $D_{8}$ if $p=2$.

Proof. (a) If $P=D_{8}$ or if $P$ is not dihedral, then there exists a normal elementary abelian subgroup $E$ of rank 2, by a well-known result of Hall, using also the fact that $m(P) \geq 2$ and that $P$ cannot be semi-dihedral because $r \geq 2$ by assumption (see Theorem 5.4.10 in [Go]). We number the representatives $\mathcal{C}_{i}$ in such a way that $E \in \mathcal{C}_{1}$. 
Assume that $m(P) \geq 3$ and let $F$ be any elementary abelian subgroup of rank $\geq 3$. Then $C_{F}(E)$ has index 1 or $p$ in $F$, because $F / C_{F}(E)$ embeds in $\operatorname{Aut}(E)$, hence $C_{F}(E) \in \mathcal{A}$. In view of the sequence of inclusions

$$
E \leq E \cdot C_{F}(E) \geq C_{F}(E) \leq F,
$$

we conclude that $E \in \mathscr{B}$. Therefore $\mathcal{C}_{1}=\mathscr{B}$, proving (a).

(b) If $P$ is a dihedral 2-group, then there are 2 conjugacy classes of maximal elementary abelian subgroups of rank 2 (none of them is normal if $|P| \geq 16)$. For any such subgroup $F$, we have $F=Z \times C$ where $Z=Z(P)$ and $|C|=2$, and moreover $C_{P}(C)=F$ and $N_{P}(F)=D_{8}$. So (b) and (c) hold in this case.

If $P$ is not dihedral, let $E$ be the normal elementary abelian subgroup of rank 2 considered in part (a). This subgroup $E$ has the following properties. First $E$ is non-central, otherwise $E$ would be contained in every maximal elementary abelian subgroup, and the poset $\mathcal{A}$ would be connected, contrary to the assumption that $r \geq 2$. Next $Z=E \cap Z(P) \neq\{1\}$ because $E$ is normal and so $|Z|=p$. Note that $Z$ is contained in every maximal elementary abelian subgroup. Finally the subgroup $M=C_{P}(E)$ is a proper subgroup of $P$, otherwise $E$ would be central. Since $N_{P}(E)=P$ and $P / M$ embeds in $\operatorname{Aut}(E), M$ has index $p$ in $P$.

Since $\mathcal{C}_{1}=\mathscr{B}$ whenever $m(P) \geq 3$, it follows that in all cases, for $2 \leq i \leq r$, we have $\mathcal{C}_{i}=\left\{E_{i}\right\}$ where $E_{i}$ is maximal elementary abelian of rank 2 . Moreover, as before, $E_{i}$ cannot be central, otherwise $\mathcal{A}$ would be connected, and therefore $E_{i}$ contains a non-central subgroup $S_{i}$ of order $p$. Also $Z \leq E_{i}$ and so $E_{i}=Z \times S_{i}$. Now $S_{i}$ does not centralize $E$, otherwise $E_{i}<E \times S_{i}$, contrary to the maximality of $E_{i}$. Therefore $S_{i} \leq M$ and since $M$ has index $p$ we have that $P=S_{i} \cdot M$. Hence $C_{P}\left(S_{i}\right)=S_{i} \cdot C_{M}\left(S_{i}\right)$, that is, $C_{P}\left(S_{i}\right)=S_{i} \times L_{i}$ where $L_{i}=C_{M}\left(S_{i}\right)$. If $A$ is a maximal elementary abelian subgroup of $L_{i}$, then $Z \leq A$ and

$$
E_{i}=Z \times S_{i} \leq A \times S_{i},
$$

hence $Z=A$ by maximality of $E_{i}$. This shows that $m\left(L_{i}\right)=1$ and therefore $L_{i}$ is cyclic or generalized quaternion (Theorem 5.4.10 in [Go]). This completes the proof of (b).

(c) Clearly $C_{P}\left(E_{i}\right)=C_{P}\left(S_{i}\right)$ because $E_{i}=Z \times S_{i}$. Since $E$ is normal and $E \cap E_{i}=Z, E \cdot E_{i}$ is a group of order $p^{3}$ and $E_{i}$ has index $p$ in $E \cdot E_{i}$. Therefore $E$ normalizes $E_{i}$. But we have seen that $E$ does not centralize $S_{i}$ and so $\left|N_{P}\left(E_{i}\right): C_{P}\left(E_{i}\right)\right| \neq 1$. Since $N_{P}\left(E_{i}\right) / C_{P}\left(E_{i}\right)$ embeds in $\operatorname{Aut}\left(E_{i}\right)$, we have $\left|N_{P}\left(E_{i}\right): C_{P}\left(E_{i}\right)\right|=p$. Moreover, since $E \cdot E_{i}$ is non-abelian of order $p^{3}$ and has two elementary abelian subgroups of rank 2 , it has to be extraspecial of exponent $p$ if $p$ is odd and isomorphic to $D_{8}$ if $p=2$. Finally, we have

$$
N_{P}\left(E_{i}\right)=E \cdot C_{P}\left(E_{i}\right)=E \cdot E_{i} \cdot L_{i}
$$

and we obtain a central product $\left(E \cdot E_{i}\right) * L_{i}$ because $\left(E \cdot E_{i}\right) \cap L_{i}=Z$. 
Remark 2.3. Note that Lemma 2.1 follows from the argument of the proof of (a) and that this argument also shows that if $m(P) \geq 3$ then every normal elementary abelian subgroup of rank 2 belongs to $\mathscr{B}$.

\section{Alperin's theorem revisited}

We begin by recalling basic definitions and establishing some notation that is of use throughout the paper. The group $T(P)$ has elements consisting of equivalence classes $[M]$ of endo-trivial modules $M$. The equivalence relation is that $[M]=[N]$ if $M \oplus Q_{1} \cong N \oplus Q_{2}$ for some projective modules $Q_{1}$ and $Q_{2}$. We use an additive notation for the group law in $T(P)$. That is the operation is given by

$$
[M]+\left[M^{\prime}\right]=\left[M \otimes M^{\prime}\right] .
$$

For a $k P$-module $M$, let $\Gamma(M)$ be the sum of all the indecomposable components of $M$ having vertex $P$. Thus, $M \cong \Gamma(M) \oplus U$, where $U$ is a direct sum of submodules with vertex properly contained in $P$. Both $\Gamma(M)$ and $U$ are well defined up to isomorphism. Let $M^{\otimes^{n}}$ denote the tensor product of $n$ copies of $M$.

An endo-permutation module is a finitely generated module $M$ whose endomorphism ring $\operatorname{Hom}_{k}(M, M)$ is a permutation module. In the Dade group $D(P)$, the elements are classes $[M]$ of endo-permutation modules $M$ having at least one indecomposable summand with vertex $P$ (unique up to isomorphism). Hence for $M$ such a module $[M]=[\Gamma(M)]$. The operation is given by

$$
[M]+[N]=[M \otimes N]=[\Gamma(M \otimes N)] .
$$

The group of endo-trivial modules $T(G)$ injects into $D(P)$ and can be considered as a subgroup of the Dade group in a natural way. In particular, we use the symbol $[M]$ to denote the class of $M$ in either $D(P)$ or $T(P)$, as appropriate. The reader should be warned that the classes do not mean the same thing in the two different groups. However, a class $[M]$ in $D(P)$ is in the image of a class in $T(P)$ if and only if the module $\Gamma(M)$ is an endo-trivial module. We emphasize also that $\Gamma(M)$ is well defined up to isomorphism by its class $[M]$ in $D(P)$.

Alperin's theorem asserts that the torsion free rank of $T(P)$ is equal to the number $r$ of conjugacy classes of connected components of the poset $\mathcal{A}$ of the previous section. The proof uses relative syzygies (see also [BoTh] for another approach using tensor induction). For later use, we need to revisit Alperin's proof in more detail, taking also into account that $T(P)$ has no torsion, by one of the main results of [CaTh2].

For any $p$-group $P$ and for any finite $P$-set $X$, we let $\Omega_{X}^{1}(k)$ denote the first relative syzygy of the trivial $k P$-module, that is, the kernel of the map $k X \rightarrow k$ mapping every element of $X$ to 1 , where $k X$ denotes the permutation module with basis $X$. We shall use the relative syzygies $\Omega_{P / Q}^{1}(k)$ for suitable subgroups $Q$. When $Q=1$, we obtain the ordinary 
syzygy $\Omega_{P}^{1}(k)$ of the trivial $k P$-module, that is, the augmentation ideal of the group algebra $k P$. Also, $\Omega_{P}^{-1}(k)$ denotes the dual of $\Omega_{P}^{1}(k)$, whose class in $T(P)$ is the inverse of the class of $\Omega_{P}^{1}(k)$. For any $k P$-module $M$, let $\Omega_{X}^{1}(M)$ denote the kernel of a relative $k X$-projective cover $U \longrightarrow M$. Then inductively, we define $\Omega_{X}^{n}(M)=\Omega_{X}^{1}\left(\Omega_{X}^{n-1}(M)\right)$. The usual calculus for these modules is that

$$
\Omega_{X}^{m}(M) \otimes \Omega_{X}^{n}(N) \cong \Omega_{X}^{m+n}(M \otimes N) \oplus W
$$

where $W$ is some relatively $X$-projective module. Note that if $X=P, W$ is a projective $k P$-module.

We select maximal elementary abelian subgroups $E_{2}, \ldots, E_{r}$ of rank 2 as in Lemma 2.2 and we let $S_{i}$ denote a non-central subgroup of $E_{i}$, for every $i=2, \ldots, r$. Moreover, we choose arbitrarily a subgroup $E_{1}$ in the remaining connected component $\mathcal{C}_{1}$. Recall that the group $T\left(E_{i}\right)$ of endotrivial modules for $E_{i}$ is isomorphic to $\mathbb{Z}$, generated by the class of $\Omega_{E_{i}}^{1}(k)$ (Dade [Da]). We define

$$
\alpha_{i}: T(P) \stackrel{\operatorname{Res}_{E_{i}}^{P}}{\longrightarrow} T\left(E_{i}\right) \stackrel{\sim}{\longrightarrow} \mathbb{Z} .
$$

That is, $\alpha_{i}([M])=n$ if $M \downarrow E_{i} \cong \Omega_{E_{i}}^{n}(k) \oplus U$ for some projective $k E_{i^{-}}$ module $U$. Let

$$
\alpha=\prod_{i=1}^{r} \alpha_{i}: T(P) \longrightarrow \prod_{i=1}^{r} \mathbb{Z}=\mathbb{Z}^{r} .
$$

Theorem 3.1. Let $P$ be a finite p-group having a maximal elementary abelian subgroup of rank 2, and suppose that $P$ is not semi-dihedral. Let $\alpha$ be defined as above.

(a) The map $\alpha$ is injective.

(b) (Alperin [Al]) The map a has a finite cokernel. In other words it is a rational isomorphism. In particular $T(P)$ is free abelian of rank $r$.

(c) The classes of the modules $\Omega_{P}^{1}(k), N_{2}, \ldots, N_{r}$ defined below form a rational basis of $\mathbb{Q} \otimes T(P)$. If $2 \leq i \leq r$, define the $k P$-module $M_{i}=\Omega_{P}^{-1}(k) \otimes \Omega_{P / S_{i}}^{1}(k)$. If $C_{P}\left(S_{i}\right) / S_{i}$ is cyclic of order $\geq 3$, then $N_{i} \cong \Gamma\left(M_{i}^{\otimes^{2}}\right)$ is endo-trivial and we have

$$
\alpha_{j}\left(\left[N_{i}\right]\right)=\left\{\begin{array}{cc}
0 & \text { if } j \neq i \\
-2 p & \text { if } j=i
\end{array}\right.
$$

If $p=2$ and $C_{P}\left(S_{i}\right) / S_{i}$ is cyclic of order 2 , then $N_{i} \cong M_{i}$ is endo-trivial and we have

$$
\alpha_{j}\left(\left[N_{i}\right]\right)=\left\{\begin{aligned}
0 & \text { if } j \neq i \\
-2 & \text { if } j=i
\end{aligned}\right.
$$


If $p=2$ and $C_{P}\left(S_{i}\right) / S_{i}$ is generalized quaternion, then $N_{i} \cong \Gamma\left(M_{i}^{\otimes^{4}}\right)$ is endo-trivial and we have

$$
\alpha_{j}\left(\left[N_{i}\right]\right)=\left\{\begin{array}{cc}
0 & \text { if } j \neq i \\
-8 & \text { if } j=i
\end{array}\right.
$$

Finally, $\alpha_{j}\left(\left[\Omega_{P}^{1}(k)\right]\right)=1$ for every $j$.

Proof. The group $P$ cannot be cyclic nor quaternion and is not semi-dihedral by assumption. Therefore, by Theorem 1.2 of [CaTh2], the restriction map

$$
\text { Res }: T(P) \longrightarrow \prod_{E} T(E) \cong \prod_{E} \mathbb{Z}
$$

is an injection, where $E$ runs through the set of all elementary abelian subgroups of $P$ of rank 2. Clearly, it suffices to choose representatives of conjugacy classes of such subgroups. This proves (a) when $m(P)=2$. Now if $m(P) \geq 3$, then there is a big component $\mathscr{B}$ and the restriction map $T(E) \longrightarrow T(F)$ is an isomorphism whenever $E, F \in \mathscr{B}$ with $F \leq E$, because both groups are isomorphic to $\mathbb{Z}$, generated by the class of $\Omega^{1}(k)$. Therefore it suffices to choose the single subgroup $E_{1} \in \mathcal{B}$ and (a) also follows in this case.

It is clear that (c) implies (b). Actually (c) is implicit in Alperin's paper $[\mathrm{Al}]$ and all the ideas come from that paper. We now indicate how to prove (c) and refer to Bouc's paper [Bo] for a detailed exposition of the machinery concerning relative syzygies. We remind the reader of the notational convention regarding the classes $[M]$ in $D(P)$ and $T(P)$, that was stated at the beginning of the section.

By Theorem 1 of [Al] or Lemma 2.3.3 of [Bo], the module $\Omega_{P / S_{i}}^{1}(k)$ is an endo-permutation module. Hence, so is $M_{i}$. In order to prove that a suitable multiple in the Dade group of $\left[\Omega_{P / S_{i}}^{1}(k)\right]$ is endo-trivial, it suffices to prove that its class in $D(P)$ lies in the kernel of the deflation-restriction map

$$
\operatorname{Def}_{N_{P}(Q) / Q}^{N_{P}(Q)} \operatorname{Res}_{N_{P}(Q)}^{P}: D(P) \longrightarrow D\left(N_{P}(Q) / Q\right)
$$

for every non-trivial subgroup $Q$ of $P$ (see 2.1.2 in $[\mathrm{Pu}]$ ). Now by Lemma 4.2.1 in [Bo],

$$
\operatorname{Def}_{N_{P}(Q) / Q}^{N_{P}(Q)} \operatorname{Res}_{N_{P}(Q)}^{P}\left(\left[\Omega_{P / S_{i}}^{1}(k)\right]\right)=\left[\Omega_{\left(P / S_{i}\right) Q}^{1}(k)\right]
$$

where $\left(P / S_{i}\right)^{Q}$ is the set of $Q$-fixed points in $P / S_{i}$, viewed as an $N_{P}(Q) / Q$ set. If $Q$ is not conjugate to $S_{i}$, then $\left(P / S_{i}\right)^{Q}$ is empty and therefore

$$
\operatorname{Def}_{N_{P}(Q) / Q}^{N_{P}(Q)} \operatorname{Res}_{N_{P}(Q)}^{P}\left(\left[\Omega_{P / S_{i}}^{1}(k)\right]\right)=0 .
$$

If $Q$ is conjugate to $S_{i}$ then we can assume that $Q=S_{i}$ and we have that

$$
\operatorname{Def}_{N_{P}\left(S_{i}\right) / S_{i}}^{N_{P}\left(S_{i}\right)} \operatorname{Res}_{N_{P}\left(S_{i}\right)}^{P}\left(\left[\Omega_{P / S_{i}}^{1}(k)\right]\right)=\left[\Omega_{N_{P}\left(S_{i}\right) / S_{i}}^{1}(k)\right] .
$$


But we have $N_{P}\left(S_{i}\right) / S_{i}=C_{P}\left(S_{i}\right) / S_{i} \cong L_{i}$ by Lemma 2.2 and $\left[\Omega_{L_{i}}^{1}(k)\right]$ has order 2 if $L_{i}$ is cyclic of order $\geq 3$, order 1 if $L_{i}$ is cyclic of order 2, and order 4 if $L_{i}$ is generalized quaternion. This shows that the corresponding multiple of $\left[\Omega_{P / S_{i}}^{1}(k)\right]$ lies in the kernel of the deflation-restriction map. Consequently, the class of the corresponding multiple of $M_{i}$ in the Dade group is also the class of an endo-trivial module.

In order to compute the image of $N_{i}$ under the map $\alpha_{j}$, we need to compute the restriction $M_{i} \downarrow_{E_{j}}^{P}$, and also $\Omega_{P / S_{i}}^{1}(k) \downarrow_{E_{j}}^{P}$. By Corollary 4.1.2 in [Bo], we have that

$$
\left[\Omega_{P / S_{i}}^{1}(k) \downarrow_{E_{j}}^{P}\right]=\left[\Omega_{\operatorname{Res}_{E_{j}}^{P}\left(P / S_{i}\right)}^{1}(k)\right]= \begin{cases}{\left[\Omega_{E_{j}}^{1}(k)\right]} & \text { if } j \neq i, \\ {\left[\Omega_{\operatorname{Res}_{E_{i}}^{P}\left(P / S_{i}\right)}^{1}(k)\right]} & \text { if } j=i .\end{cases}
$$

Now the Mackey decomposition shows that $\operatorname{Res}_{E_{i}}^{P}\left(P / S_{i}\right)$ is the disjoint union of a free $E_{i}$-set and of $\left|C_{P}\left(E_{i}\right): E_{i}\right|$ copies of

$$
\bigsqcup_{g \in\left[N_{P}\left(E_{i}\right) / C_{P}\left(E_{i}\right)\right]} E_{i} / S_{i}^{g}=\bigsqcup_{s=1}^{p} E_{i} / S_{i}^{g_{s}},
$$

where $g_{1}, \ldots, g_{p}$ are representatives of $N_{P}\left(E_{i}\right) / C_{P}\left(E_{i}\right)$ (see Lemma 2.2). By Lemma 3.2.7 in [Bo], we deduce that

$$
\left[\Omega_{P / S_{i}}^{1}(k) \downarrow_{E_{i}}^{P}\right]=\left[\Omega_{\bigsqcup_{s=1}^{p} E_{i} / S_{i}^{g s}}^{1}(k)\right] .
$$

Now by repeated applications of Lemma 5.2.1 of [Bo] (which is also the method used on pp. 9-10 of [Al]), we obtain

$$
\left[\Omega_{\bigsqcup_{s=1}^{p} E_{i} / S_{i}^{g_{s}}}^{1}(k)\right]=-(p-1)\left[\Omega_{E_{i}}\right]+\sum_{s=1}^{p}\left[\Omega_{E_{i} / S_{i}^{g_{s}}}^{1}(k)\right] .
$$

But $E_{i} / S_{i}^{g_{s}}$ is cyclic of order $p$, so $\left[\Omega_{E_{i} / S_{i}^{g_{s}}}^{1}(k)\right]=0$ if $p=2$ and $2\left[\Omega_{E_{i} / S_{i}^{g s}}^{1}(k)\right]=0$ if $p$ is odd. This shows that

$$
\alpha_{j}\left(\left[\Gamma\left(\Omega_{P / S_{i}}^{1}(k)^{\otimes^{m}}\right)\right]\right)= \begin{cases}m & \text { if } j \neq i, \\ -m(p-1) & \text { if } j=i,\end{cases}
$$

where we take $m=1$ (resp. 2, resp. 4) if $C_{P}\left(S_{i}\right) / S_{i} \cong L_{i}$ is cyclic of order 2 (resp. cyclic of order $\geq 3$, resp. generalized quaternion). The class $\left[N_{i}\right]$ is now obtained by adding $\left.\left[\Omega_{P}^{-1}(k)^{\otimes^{m}}\right]=m\left[\Omega_{P}^{-1}(k)\right]\right)$, whose image under $\alpha$ is $(-m,-m, \ldots,-m)$. The result of the computation for $\alpha_{j}\left(\left[N_{i}\right]\right)$ follows. 
Theorem 3.1 raises the question of describing explicitly the image of $\alpha$ and this is the main purpose of the present paper. We prove that the basis appearing in Theorem 3.1 is actually an integral basis of $T(P)$.

Note that we can assume that the integer $r$ is at least 2 , for if $r=1$ then $\operatorname{Res}_{E_{1}}^{P}: T(P) \longrightarrow T\left(E_{1}\right) \cong \mathbb{Z}$ is an isomorphism and $T(P)$ is generated by the class of the ordinary syzygy $\Omega_{P}^{1}(k)$. One of the effects of the assumption that $r \geq 2$ is that all $E_{i}$ 's intersect in the subgroup $Z$ of order $p$ considered in Sect. 2, namely the unique central subgroup of order $p$. This motivates the situation considered in the next two sections, where $Z$ will denote a central subgroup of order $p$.

Remark 3.2. In the first case of (c), the module $N_{i}$ has the form of a translate of a relative syzygy, $N_{i} \cong \Omega^{-2}\left(\Omega_{P / S_{i}}^{2}(k)\right)$. Likewise, in the third case, that $p=2$ and $C_{P}\left(S_{i}\right) / S_{i}$ is generalized quaternion, we have that $N_{i} \cong$ $\Omega^{-4}\left(\Omega_{P / S_{i}}^{4}(k)\right)$. That is, in all cases, the module $N_{i}$ is a translate of a relative syzygy of the trivial module.

\section{Decomposing endo-trivial modules}

The purpose of this section is to show that Theorem 5.6 of [CaTh2] is still valid under different assumptions. The hypotheses in [CaTh2] is about a critical endo-trivial module, but it was shown later in the paper that no such module exists. So the result needs to be revisited in order to be used in a different context.

Throughout this section, we let $P$ be a $p$-group, $Z$ be a central subgroup of order $p$ generated by $z$, and we write $\bar{P}=P / Z$. Let $M$ be a $k P$-module such that $M \downarrow_{Z}^{P} \cong k \oplus$ (free). Recall that projective modules are free because $P$ is a $p$-group. We define $M^{\prime}=\left\{m \in M \mid(z-1)^{p-1} m=0\right\}$ and we set

$$
\bar{M}=M / M^{\prime} \text {. }
$$

Since $(z-1) \bar{M}=0$, the module $\bar{M}$ can be viewed as a $k \bar{P}$-module. Moreover, by Lemma 5.2 in [CaTh2], we have $\operatorname{Dim}(M)=p \operatorname{Dim}(\bar{M})+1$ and

$$
\bar{M} \cong(z-1)^{i} M /(z-1)^{i+1} M \cong(z-1)^{p-1} M, \quad(i=1, \ldots, p-1) .
$$

In fact, $(z-1) M$ is free as a module over $k Z /(z-1)^{p-1}$.

Lemma 4.1. Let $M$ be a $k P$-module such that $M \downarrow_{Z}^{P} \cong k \oplus$ (free). Then $M \cong k \oplus$ (free) as a $k P$-module if and only if $\bar{M}$ is a free $k \bar{P}$-module. More precisely, $M$ has a free summand with $r$ generators as a $k P$-module if and only if $\bar{M}$ has a free summand with $r$ generators as a $k \bar{P}$-module. In particular, if $M$ is indecomposable, then $\bar{M}$ has no projective summands.

Proof. This is Lemma 5.3 in [CaTh2]. The assumption in [CaTh2] is slightly different but the proof can be applied without change. 
We refer to [CaTh2] for the definition and properties of the variety $V_{\bar{P}}(\bar{L})$ attached to any $k \bar{P}$-module $\bar{L}$.

Theorem 4.2. Let $M$ be a $k P$-module such that $M \downarrow Z P \cong \oplus$ (free). Suppose that $\bar{M}=\bar{M}_{1} \oplus \bar{M}_{2}$ where $\bar{M}_{1}$ and $\bar{M}_{2}$ are $k \bar{P}$-submodules such that the varieties satisfy

$$
V_{\bar{P}}\left(\bar{M}_{1}\right) \cap V_{\bar{P}}\left(\bar{M}_{2}\right)=\{0\} .
$$

(a) There exist $k P$-modules $N_{1}$ and $N_{2}$ such that $N_{i} \downarrow_{Z}^{P} \cong k \oplus$ (free) and $\bar{N}_{i} \cong \bar{M}_{i}$ for $1 \leq i \leq 2$.

(b) If moreover $\bar{M}_{2}$ is a free $k \bar{P}$-module, then there exists a submodule $N_{1}$ of $M$ satisfying the conditions of (a) and such that $M=N_{1} \oplus$ (free).

Proof. (a) The result is similar to Theorem 5.6 in [CaTh2] and the proof is almost identical. Therefore we only indicate where the proof needs to be adapted. The construction of $N_{1}$ and $N_{2}$ is the same and we use the same notation. We have $(z-1) M=W_{1} \oplus W_{2}$ with $W_{i} /(z-1) W_{i} \cong \bar{M}_{i}$ for $i=1,2$ (and it is here that the condition on the varieties is used). We let $M_{i} \subseteq M$ be the inverse image of $\bar{M}_{i}$ under the quotient map $M \longrightarrow$ $M / M^{\prime}=\bar{M}$ and we define $N_{1}=M_{1} / W_{2}$ and $N_{2}=M_{2} / W_{1}$. Then we have $\operatorname{Dim}\left(N_{i}\right)=p \operatorname{Dim}\left(\bar{M}_{i}\right)+1$.

Next we prove that $N_{1} \downarrow_{Z}^{P} \cong k \oplus$ (free) (by an argument similar to, but much easier than the one used in [CaTh2]). We have $(z-1)^{p-1} N_{1}$ $\cong(z-1)^{p-1} M_{1}$ because $N_{1}=M_{1} / W_{2}$ and $(z-1)^{p-1} W_{2}=0$. Now $(z-1)^{p-1} M_{1} \cong \bar{M}_{1}$ because multiplication by $(z-1)^{p-1}$ induces an isomorphism $\bar{M} \cong(z-1)^{p-1} M$. Therefore

$$
\left(\sum_{i=0}^{p-1} z^{i}\right) N_{1}=(z-1)^{p-1} N_{1} \cong \bar{M}_{1}
$$

and it follows that $N_{1} \downarrow_{Z}^{P}$ has a free submodule of dimension $p \operatorname{Dim}\left(\bar{M}_{1}\right)$, that is, $\operatorname{Dim}\left(N_{1}\right)-1$. The only way this can happen is if $N_{1} \downarrow_{Z}^{P} \cong k \oplus$ (free).

Finally we have to show that $\bar{N}_{1} \cong \bar{M}_{1}$, and similarly for $N_{2}$. The proof is exactly the same as in [CaTh2]: $\bar{N}_{1}$ appears naturally as a quotient of $\bar{M}_{1}$ and they have the same dimension.

(b) This additional part, which was not proved in [CaTh2], is based on the construction of $N_{1}$. Let $\bar{x}_{1}, \ldots, \bar{x}_{m}$ be a basis of $\bar{M}_{2}$ as a $k \bar{P}$-module, let $x_{i} \in M_{2}$ be a lift of $\bar{x}_{i}$ and let $F$ be the $k P$-submodule of $M_{2}$ generated by $x_{1}, \ldots, x_{m}$. By Lemma $4.1, F$ is free of dimension $m|P|=p m|\bar{P}|=$ $p \operatorname{Dim}\left(\bar{M}_{2}\right)$. Now

$$
(z-1)^{p-1} F \subseteq(z-1)^{p-1} M_{2}=(z-1)^{p-2} W_{2} \cong \bar{M}_{2} \cong F /(z-1) F,
$$

and therefore $(z-1)^{p-1} F=(z-1)^{p-2} W_{2}$. It follows that $W_{1} \cap F=\{0\}$ because if we choose $w \in W_{1} \cap F$, then we can assume that $(z-1) w=0$ 
(replacing $w$ by $(z-1)^{i} w$ for some $i$ ), so that $w \in(z-1)^{p-1} F$ which intersects $W_{1}$ trivially. Hence $W_{1} \cap(z-1) F=\{0\} . \operatorname{But} \operatorname{Dim}(F)=p \operatorname{Dim}\left(\bar{M}_{2}\right)=$ $p \operatorname{Dim}\left((z-1)^{p-2} W_{2}\right)$, hence

$$
\operatorname{Dim}((z-1) F)=(p-1) \operatorname{Dim}\left((z-1)^{p-2} W_{2}\right)=\operatorname{Dim}\left(W_{2}\right) .
$$

Since $(z-1) M=W_{1} \oplus W_{2}$, we must also have $(z-1) M=W_{1} \oplus(z-1) F$ and so we can choose $W_{2}=(z-1) F$ for the construction of $N_{1}=M_{1} / W_{2}$.

Since $F \subseteq M_{2}$, we have $M_{1} \cap F=M_{1} \cap M_{2} \cap F=M^{\prime} \cap F$. But the elements of $F$ lying in the kernel of multiplication by $(z-1)^{p-1}$ are precisely the multiples of $(z-1)$ and therefore $M^{\prime} \cap F=(z-1) F=W_{2}$. Hence $M_{1} \cap F=W_{2}$. On the other hand, since $M^{\prime} \subseteq M_{1}$, we have

$$
M_{1}+F=M_{1}+M^{\prime}+F=M_{1}+M_{2}=M .
$$

It follows that

$$
M / F=\left(M_{1}+F\right) / F \cong M_{1} /\left(M_{1} \cap F\right)=M_{1} / W_{2}=N_{1} .
$$

Since $F$ is free, hence injective (because $k P$ is self-injective), $F$ is a direct summand of $M$ and we obtain $M=F \oplus N_{1}^{\prime}$, with $N_{1}^{\prime}$ isomorphic to $N_{1}$, as required.

Now we apply this theorem to the case of an endo-trivial module over a $p$-group $P$ having a maximal elementary abelian subgroup of rank 2 . We wish to prove that the modules $N_{1}$ and $N_{2}$ are again endo-trivial in this case and that we have precise information about their restrictions to elementary abelian subgroups.

Let $E_{1}, \ldots, E_{r}$ be elementary abelian subgroups chosen as in Theorem 3.1. If $M$ is any endo-trivial $k P$-module, then $\operatorname{Res}_{E_{1}}^{P}[M]=\left[\Omega_{E_{1}}^{s}(k)\right]$ for some $s$, where $[M]$ denotes the class of $M$ in the group $T(P)$ of endotrivial $k P$-modules. Therefore

$$
\operatorname{Res}_{E_{1}}^{P}\left[\Omega_{P}^{-s}(M)\right]=\operatorname{Res}_{E_{1}}^{P}\left[\Omega_{P}^{-s}(k) \otimes M\right]=\left[\Omega_{E_{1}}^{-s}(k) \otimes \Omega_{E_{1}}^{s}(k)\right]=[k] .
$$

It follows that, up to a shift, we can always choose $M$ such that $\operatorname{Res}_{E_{1}}^{P}[M]$ is trivial, that is, $M \downarrow_{E_{1}}^{P} \cong k \oplus$ (free).

As noticed at the end of Sect. 2, we can assume that $r \geq 2$ and therefore all $E_{i}$ 's intersect in a central subgroup $Z$ of order $p$. In particular $M \downarrow_{Z}^{P} \cong$ $k \oplus$ (free) and we can use Theorem 4.2.

Theorem 4.3. Let $P$ be a p-group having a maximal elementary abelian subgroup of rank 2 and let $E_{1}, \ldots, E_{r}$ be elementary abelian subgroups chosen as in Theorem 3.1. We assume that $r \geq 2$ and we let $Z$ be the unique central subgroup of order $p$ (contained in every $E_{j}$ ). Let $M$ be an endo-trivial $k P$-module such that $M \downarrow_{E_{1}}^{P} \cong k \oplus$ (free). Suppose that $\bar{M}=\bar{M}_{1} \oplus \bar{M}_{2}$ where $\bar{M}_{1}$ and $\bar{M}_{2}$ are $k \bar{P}$-submodules such that their varieties $V_{1}=V_{\bar{P}}\left(\bar{M}_{1}\right)$ and $V_{2}=V_{\bar{P}}\left(\bar{M}_{2}\right)$ satisfy

$$
V_{1} \cap V_{2}=\{0\} .
$$


(a) There exist endo-trivial kP-modules $N_{1}$ and $N_{2}$ such that $N_{i} \downarrow_{Z}^{P} \cong$ $k \oplus$ (free) and $\bar{N}_{i} \cong \bar{M}_{i}$ for $1 \leq i \leq 2$.

(b) For $E=E_{1}$ and for any elementary abelian subgroup $E$ lying in the big component, we have $N_{i} \downarrow_{E}^{P} \cong k \oplus$ (free) for $1 \leq i \leq 2$.

(c) For $E=E_{j}$ where $j=2, \ldots, r$, one of the two modules $N_{1}, N_{2}$ is trivial on restriction to $E$. More precisely, setting $\bar{E}=E / Z$, we have the following cases:

(1) $V_{\bar{E}}(\bar{M})=\{0\}$ in which case $N_{i} \downarrow_{E}^{P} \cong k \oplus$ (free) for $1 \leq i \leq 2$;

(2) $V_{\bar{E}}(\bar{M})=V_{\bar{E}}(k)$ and $\operatorname{res}_{\bar{P}}^{*} \bar{E}\left(V_{\bar{E}}(k)\right) \subseteq V_{1}$ in which case $V_{\bar{E}}\left(\bar{N}_{1}\right)=$ $V_{\bar{E}}(k), M \downarrow_{E}^{P} \cong N_{1} \downarrow_{E}^{P} \oplus$ (free), and $N_{2} \downarrow_{E}^{P} \cong k \oplus$ (free);

(3) $V_{\bar{E}}(\bar{M})=V_{\bar{E}}(k)$ and $\operatorname{res}_{\bar{P}}^{*}, \bar{E}\left(V_{\bar{E}}(k)\right) \subseteq V_{2}$ in which case $V_{\bar{E}}\left(\bar{N}_{2}\right)=$ $V_{\bar{E}}(k), M \downarrow_{E}^{P} \cong N_{2} \downarrow_{E}^{P} \oplus$ (free), and $N_{1} \downarrow_{E}^{P} \cong k \oplus$ (free).

Proof. (a) We use Theorem 4.2 for the existence of $N_{1}$ and $N_{2}$ and moreover we have now to prove that $N_{1}$ and $N_{2}$ are endo-trivial. This will follow if we show that they are endo-trivial on restriction to every elementary abelian subgroup of $P$, by Lemma 2.9 of [CaTh1]. Thus it suffices to prove (b) and (c), because (b) and (c) show that $N_{i} \downarrow_{E}^{P}$ is isomorphic, up to addition or deletion of free summands, either to $k$ or to $M \downarrow_{E}^{P}$, for every maximal elementary subgroup $E$ of $P$.

(b) Since $M \downarrow_{E_{1}}^{P} \cong k \oplus$ (free) by assumption, we also have $M \downarrow_{E}^{P} \cong$ $k \oplus$ (free) for any elementary abelian subgroup $E$ lying in the big component (see the beginning of the proof of Theorem 3.1). Thus $\bar{M} \downarrow \frac{\bar{P}}{E}$ is free by Lemma 4.1. Therefore $\bar{M}_{1} \downarrow \frac{\bar{P}}{E}$ and $\bar{M}_{2} \downarrow \frac{\bar{P}}{E}$ are free and so $N_{1} \downarrow_{E}^{P} \cong k \oplus$ (free) and $N_{2} \downarrow_{E}^{P} \cong k \oplus$ (free), by Lemma 4.1 again.

(c) If $V_{\bar{E}}(\bar{M})=\{0\}$, then $\bar{M} \downarrow \frac{\bar{P}}{E}$ is free and we conclude as in case (b). Since $E$ has rank 2, $\bar{E}$ is cyclic of order $p$ and the only other possibility is $V_{\bar{E}}(\bar{M})=V_{\bar{E}}(k)$, which is a line. Then $\operatorname{res}_{\bar{P}}^{*} \bar{E}\left(V_{\bar{E}}(k)\right)$ must be contained either in $V_{1}$ or in $V_{2}$. We can assume that res $\frac{*}{P} \bar{E}\left(V_{\bar{E}}(k)\right) \subseteq V_{1}$ and we must show that case (2) occurs.

Since res $\frac{*}{\bar{P}} \bar{E}\left(V_{\bar{E}}(k)\right) \subseteq V_{1}$, we have res $\frac{*}{\bar{P}} \bar{E}\left(V_{\bar{E}}(k)\right) \cap V_{2}=\{0\}$ and since $V_{2}=V_{\bar{P}}\left(\bar{N}_{2}\right)$, we must have $V_{\bar{E}}\left(\bar{N}_{2} \downarrow \frac{\bar{P}}{E}\right)=\{0\}$. Therefore $\bar{N}_{2} \downarrow \frac{\bar{P}}{E}$ is free and $N_{2} \downarrow_{E}^{P} \cong k \oplus$ (free) by Lemma 4.1. On the other hand, part (b) of Theorem 4.2 applied to $M \downarrow_{E}^{P}$ shows that $M \downarrow_{E}^{P} \cong N_{1} \downarrow_{E}^{P} \oplus$ (free).

\section{Tensoring endo-trivial modules}

In this section we show that Theorem 5.7 of [CaTh2] is still valid under different assumptions. As in the previous section, the result needs to be revisited in order to be used in a different context. However, there is only a cosmetic change to be made. We assume again that $P$ is a $p$-group with a central subgroup $Z$ of order $p$. 
Theorem 5.1. Let $M_{1}$ and $M_{2}$ be indecomposable endo-trivial $k P$-modules such that $\left(M_{j}\right) \downarrow_{Z}^{P} \cong k \oplus$ (free) for $j=1$, 2. Suppose that the varieties of $\bar{M}_{1}$ and $\bar{M}_{2}$ satisfy

$$
V_{\bar{P}}\left(\bar{M}_{1}\right) \cap V_{\bar{P}}\left(\bar{M}_{2}\right)=\{0\} .
$$

Then $M_{1} \otimes M_{2} \cong M \oplus$ (free) where $M$ is an indecomposable endo-trivial $k P$-module such that $M \downarrow Z P \cong \oplus$ (free) and $\bar{M} \cong \bar{M}_{1} \oplus \bar{M}_{2}$.

Proof. The proof is exactly the same as in Theorem 5.7 of [CaTh2], except for the following minor changes. In [CaTh2], the modules $M_{1}$ and $M_{2}$ were assumed to be critical and then $M$ was shown to be critical. Here we only assume that $M_{j}$ is indecomposable endo-trivial such that $\left(M_{j}\right) \downarrow_{Z}^{P} \cong$ $k \oplus$ (free) for $j=1,2$ and we only have to show that $M$ is indecomposable endo-trivial such that $M \downarrow_{Z}^{P} \cong k \oplus$ (free). The new assumption has no effect on the proof and therefore only the new aspect of the conclusion needs to be proved.

Explicitly, the proof that $M_{1} \otimes M_{2} \cong M \oplus$ (free) applies without change and so does the proof that $\bar{M} \cong \bar{M}_{1} \oplus \bar{M}_{2}$. In order to prove the new conclusion, it suffices to notice that if $M_{1}$ and $M_{2}$ are endo-trivial then so are $M_{1} \otimes M_{2}$ and $M$, and moreover if $\left(M_{j}\right) \downarrow_{Z}^{P} \cong k \oplus$ (free) for $j=1,2$, then $\left(M_{1} \otimes M_{2}\right) \downarrow_{Z}^{P} \cong k \oplus$ (free), and hence $M \downarrow_{Z}^{P} \cong k \oplus$ (free). Since $M_{j}$ is indecomposable, the $k \bar{P}$-module $\bar{M}_{j}$ does not have free summands by Lemma 4.1. Thus $\bar{M}$ has no free summand and it follows that $M$ has no free summand by Lemma 4.1 again. This shows that $M$ is indecomposable.

\section{Endo-trivial modules for small extraspecial groups}

In this section we describe explicitly all endo-trivial modules for a $p$-group $P$ which is either extraspecial or almost extraspecial. We refer to [Go] for extraspecial groups and [CaTh2] for almost extraspecial groups. If every maximal elementary abelian subgroup has rank $\geq 3$, then $T(P) \cong \mathbb{Z}$ by [CaTh2] and the only indecomposable endo-trivial modules are the syzygies $\Omega_{P}^{s}(k)$ with $s \in \mathbb{Z}$. So we can assume that there is at least one maximal elementary abelian subgroup of rank 2 and this forces $P$ to be one of the following groups:

(1) $P$ is extraspecial of order $p^{3}$ and exponent $p$ with $p$ odd. Here $P$ has $p+1$ maximal subgroups, which are elementary abelian of rank 2 .

(2) $P$ is extraspecial of order $p^{3}$ and exponent $p^{2}$ with $p$ odd. Here $P$ has a unique maximal elementary abelian subgroup of rank 2 . Therefore this case is of no interest for us since we know that $T(P) \cong \mathbb{Z}$, generated by $\Omega_{P}^{1}(k)$. Actually, $P$ is metacyclic and so this case is also covered by the work of Mazza [Ma].

(3) $P$ is almost extraspecial of order $p^{4}$ with $p$ odd, that is, $P=Q * C_{p^{2}}$, where $Q$ is extraspecial of order $p^{3}$ and exponent $p$ and $C_{p^{2}}$ is cyclic 
of order $p^{2}$. Here $P$ has $p+1$ maximal elementary abelian subgroups of rank 2.

(4) $p=2$ and $P=D_{8}$ is the dihedral group of order 8 . This case has been described in detail in [CaTh1]. There are two non-central subgroups $S_{1}$ and $S_{2}$ of order 2, up to conjugacy, and $T(P)$ is free abelian of rank 2, generated by the classes of $\Omega_{P}^{1}(k)$ and $\Omega_{P / S_{i}}^{1}(k)$ where $i$ is either 1 or 2 .

(5) $p=2$ and $P=D_{8} * Q_{8}$, the central product of the dihedral group of order 8 and the quaternion group of order 8 (so $P$ is extraspecial of order 32). Here $P$ has 5 maximal elementary abelian subgroups of rank 2.

(6) $p=2$ and $P$ is almost extraspecial of order 16 , that is, $P=D_{8} * C_{4}$, the central product of the dihedral group of order 8 and the cyclic group of order 4 . Here $P$ has 3 maximal elementary abelian subgroups of rank 2 .

Note that the list is complete because the next cases are the two extraspecial groups of order $p^{5}$ with $p$ odd and the group $D_{8} * D_{8}$ of order 32, whose maximal elementary abelian subgroups have rank at least 3 . All other extraspecial and almost extraspecial groups contain one of these groups of order $p^{5}$ and therefore do not have maximal elementary abelian subgroups of rank 2 .

We are going to treat case (1) in detail and then case (3) will follow easily. Cases (5) and (6) will be handled next.

Theorem 6.1. Let $p$ be an odd prime and let $P$ be the extraspecial group of order $p^{3}$ and exponent $p$. Let $E_{1}, \ldots, E_{p+1}$ be the maximal subgroups of $P$ (which are elementary abelian of rank 2) and for each $i$ let $S_{i}$ be a non-central subgroup of order $p$ in $E_{i}$. Then the group $T(P)$ is free abelian generated by the classes of the modules $\Omega_{P}^{1}(k), N_{2}, \ldots, N_{p+1}$, where $N_{i}=\Gamma\left(\left(\Omega_{P}^{-1}(k) \otimes \Omega_{P / S_{i}}^{1}(k)\right)^{\otimes^{2}}\right)$ for every $i=2, \ldots, p+1$.

Proof. Consider the injective map $\alpha: T(P) \longrightarrow \mathbb{Z}^{p+1}$ of Theorem 3.1. We know that $\alpha\left(\left[\Omega_{P}^{1}(k)\right]\right)=(1,1, \ldots, 1)$ and $\alpha\left(\left[N_{i}\right]\right)=-2 p \cdot e_{i}$, where $e_{i}$ denotes the $i$-th element of the canonical basis of $\mathbb{Z}^{p+1}$ (for every $i=$ $2, \ldots, p+1)$. We have to prove that any class of endo-trivial modules is a linear combination of these elements.

Let $M$ be any endo-trivial $k P$-module. By subtracting a multiple of $\left[\Omega_{P}^{1}(k)\right]$, we can assume that $\alpha_{1}([M])=0$ and we then have to prove that $[M]$ is a linear combination of $\left[N_{2}\right], \ldots,\left[N_{p+1}\right]$. In other words

$$
\alpha([M])=\left(0, s_{2}, s_{3}, \ldots, s_{p+1}\right)
$$

and we have to prove that $s_{i}$ is a multiple of $2 p$ for every $i=2, \ldots, p+1$. We proceed by induction on the number of non-zero components in $\alpha([M])$. We can assume that $M$ is indecomposable by deleting all free summands of $M$.

Suppose first that $\alpha([M])$ has a unique non-zero component $s_{q}$ where $q$ is some fixed integer with $2 \leq q \leq p+1$, and write $s=s_{q}$ for simplicity. 
By subtracting a multiple of $\left[N_{q}\right]$, we can assume that $0<s \leq 2 p$ and we now have to prove that $s=2 p$. Since $s_{1}=\alpha_{1}([M])=0,\left[M \downarrow_{E_{1}}^{P}\right]=0$, hence $M \downarrow_{E_{1}}^{P} \cong k \oplus($ free $)$ and $\operatorname{Dim}(M) \equiv 1\left(\bmod p^{2}\right)$. Now $\alpha_{q}([M])=s$, that is,

$$
M \downarrow_{E_{q}}^{P} \cong \Omega_{E_{q}}^{1}(k)^{\otimes^{s}} \oplus \text { (free) } .
$$

Because $\Omega_{E_{q}}^{1}(k)$ has dimension $p^{2}-1$, we have that $\operatorname{Dim}(M) \equiv(-1)^{s}$ $\left(\bmod p^{2}\right)$ and it follows that $s$ is even.

Now we claim that the automorphism group of $P$ acts transitively on the set of maximal subgroups of $P$. By Lemma 6.3 in [CaTh2], the subgroup of $\operatorname{Out}(P)$ fixing $Z(P)$ pointwise is isomorphic to $S p_{2}\left(\mathbb{F}_{p}\right)=S L_{2}\left(\mathbb{F}_{p}\right)$. This acts transitively on the lines of $\left(\mathbb{F}_{p}\right)^{2} \cong \bar{P}$, which correspond to the maximal subgroups of $P$. If now $\beta_{i}$ is an automorphism of $P$ mapping $E_{q}$ to $E_{i}$, then the conjugate module $L_{i}=M^{\beta_{i}}$ satisfies

$$
\alpha_{j}\left(\left[L_{i}\right]\right)= \begin{cases}s & \text { if } j=i \\ 0 & \text { otherwise }\end{cases}
$$

Since $M$ is indecomposable, so is $L_{i}$ for each $i$. By repeated applications of Theorem 5.1, we get that

$$
L_{1} \otimes L_{2} \otimes \ldots \otimes L_{p+1}=U \oplus(\text { free })
$$

where $U$ is an indecomposable endo-trivial module such that, with the notation of Sect. 4,

$$
\bar{U}=\bar{L}_{1} \oplus \bar{L}_{2} \oplus \ldots \oplus \bar{L}_{p+1} .
$$

Now for all $j$ we have that

$\alpha_{j}([U])=\alpha_{j}\left(\left[L_{1} \otimes L_{2} \otimes \ldots \otimes L_{p+1}\right]\right)=\alpha_{j}\left(\left[L_{1}\right]+\left[L_{2}\right]+\ldots+\left[L_{p+1}\right]\right)=s$,

and

$$
\alpha_{j}\left(\left[\Omega_{P}^{s}(k)\right]\right)=\alpha_{j}\left(\left[\Omega_{P}^{1}(k)^{\otimes^{s}}\right]\right)=\alpha_{j}\left(s\left[\Omega_{P}^{1}(k)\right]\right)=s .
$$

Therefore $[U]=\left[\Omega_{P}^{s}(k)\right]$ by injectivity of $\alpha$ and so $U \cong \Omega_{P}^{s}(k)$ because both modules are indecomposable. Since $\bar{M} \downarrow \frac{\bar{P}}{E_{1}}$ is free, $\operatorname{Dim}(\bar{M})=m p$ for some $m$ (hence $\left.\operatorname{Dim}(M)=m p^{2}+1\right)$. It follows that $\operatorname{Dim}(\bar{U})=(p+1) m p$, hence

$$
\operatorname{Dim}\left(\Omega_{P}^{s}(k)\right)=\operatorname{Dim}(U)=(p+1) m p^{2}+1 .
$$

But the mod- $p$ cohomology of $P$ is known (see Sect. 4 in [CaTh2]) and hence also the dimensions of the syzygies of $k$. Explicitly, by Corollary 4.4 in [CaTh2] and its proof, we have

$$
\operatorname{Dim}\left(\Omega_{P}^{2 j}(k)\right)=(j+1) p^{3}+1 \quad \text { if } 2 \leq j \leq p .
$$


Remembering that $s$ is even, we write $s=2 j$ and we obtain

$$
(p+1) m p^{2}+1=(j+1) p^{3}+1,
$$

hence $(p+1) m=(j+1) p$. Therefore $p$ divides $m$ and the only way this can happen is if $j=p$ (and $m=p$ ). This proves that $s=2 p$, as required.

Suppose now that the number of non-zero components in $\alpha([M])$ is at least 2. Because $\alpha_{1}([M])=0$, we have that $M \downarrow_{E_{1}}^{P} \cong k \oplus$ (free), and we are in a situation where we can apply Theorem 4.3. Since $M$ is indecomposable, the $k \bar{P}$-module $\bar{M}$ has no free summands (Lemma 4.1). However, $\bar{M}$ is free on restriction to $\overline{E_{1}}$, because $M \downarrow_{E_{1}}^{P} \cong k \oplus$ (free), and since $E_{1}$ is a maximal subgroup of $P$ we deduce that $\bar{M}$ is a periodic $k \bar{P}$-module (see Lemma 5.3 in [CaTh2] for details).

We use standard properties of varieties, as listed for instance in Theorem 2.2 of [CaTh2]. Since $\bar{M}$ is periodic, the variety of $\bar{M}$ is a union of lines. Let $\ell$ be one of these lines and let $W$ be the union of all the others, so that

$$
V_{\bar{P}}(\bar{M})=\ell \cup W, \quad \ell \cap W=\{0\} .
$$

Then $\bar{M}=\bar{M}_{1} \oplus \bar{M}_{2}$ with $V_{\bar{P}}\left(\bar{M}_{1}\right)=\ell$ and $V_{\bar{P}}\left(\bar{M}_{2}\right)=W$. By Theorem 4.3, there exist endo-trivial modules $N_{1}$ and $N_{2}$ satisfying the properties of that theorem. Let us write $L=N_{1}$ for simplicity. In particular we have $V_{\bar{P}}(\bar{L})=\ell$ and

$$
L \downarrow_{E_{1}}^{P} \cong k \oplus(\text { free }), \quad V_{\overline{E_{1}}}(\bar{L})=\{0\} .
$$

So for $j=2, \ldots, p+1$, we have either

$$
L \downarrow_{E_{j}}^{P} \cong k \oplus(\text { free }), \quad V_{\overline{E_{j}}}(\bar{L})=\{0\}, \quad \operatorname{res}_{\bar{P}, \overline{E_{j}}}^{*}\left(V_{\overline{E_{j}}}(k)\right) \subseteq W,
$$

or

$$
L \downarrow_{E_{j}}^{P} ¥ k \oplus(\text { free }), \quad V_{\overline{E_{j}}}(\bar{L})=V_{\overline{E_{j}}}(k), \quad \operatorname{res} \frac{*}{\bar{P}, \overline{E_{j}}}\left(V_{\overline{E_{j}}}(k)\right) \subseteq \ell .
$$

We cannot have that $L \downarrow_{E_{j}}^{P} \cong k \oplus$ (free) for all $j$, because otherwise $\alpha([L])=0$ and $L \cong k \oplus$ (free) by injectivity of $\alpha$. However, this would imply that $\bar{L}$ is free (Lemma 4.1) and $V_{\bar{P}}(\bar{L})=\{0\}$, a contradiction. Without loss of generality, we can assume that for the index $j=2$ we have

$$
L \downarrow_{E_{2}}^{P} ¥ k \oplus(\text { free }), \quad V_{\overline{E_{2}}}(\bar{L})=V_{\overline{E_{2}}}(k), \quad \operatorname{res} \bar{P}_{\bar{P}}^{*} \bar{E}_{2}\left(V_{\overline{E_{2}}}(k)\right) \subseteq \ell .
$$

Actually the latter inclusion is an equality because $\bar{P}$ is elementary abelian and res $\frac{*}{P}, \overline{E_{2}}\left(V_{\overline{E_{2}}}(k)\right)$ is a line in the plane $V_{\bar{P}}(k)$. Moreover, Theorem 4.3 also asserts that $M \downarrow_{E_{2}}^{P} \cong L \downarrow_{E_{2}}^{P} \oplus$ (free) and therefore $\alpha_{2}([L])=\alpha_{2}([M])=s_{2}$. 
Now if $j \geq 3$, res ${ }_{\bar{P}}^{*}, \overline{E_{j}}\left(V_{\overline{E_{j}}}(k)\right)$ is a line different from $\ell$ and therefore the other case occurs, namely $L \downarrow_{E_{j}}^{P} \cong k \oplus$ (free). Thus we have proved that

$$
\alpha_{j}([L])= \begin{cases}s_{2} & \text { if } j=2 \\ 0 & \text { otherwise }\end{cases}
$$

By the first step of induction, we know that $s_{2}$ is a multiple of $2 p$.

Now let $M^{\prime}$ be an indecomposable endo-trivial module in the class of $[M]-[L]$. Then $\alpha\left(\left[M^{\prime}\right]\right)$ has fewer non-zero components than $\alpha([M])$ and so they are all multiples of $2 p$ by induction. This shows that $s_{j}$ is a multiple of $2 p$ for $j \geq 3$ and completes the proof.

The following corollary is not actually needed for the proof of the main theorem. However, we include a proof for the sake of completeness.

Corollary 6.2. Let $P=Q * C_{p^{2}}$ be an almost extraspecial of order $p^{4}$ with $p$ odd, where $Q$ is extraspecial of order $p^{3}$ and exponent $p$ and $C_{p^{2}}$ is cyclic of order $p^{2}$.

(a) $\operatorname{Res}_{Q}^{P}: T(P) \longrightarrow T(Q)$ is an isomorphism.

(b) Let $E_{1}, \ldots, E_{p+1}$ be the maximal subgroups of $Q$ (which are elementary abelian of rank 2) and for each $i$ let $S_{i}$ be a non-central subgroup of order $p$ in $E_{i}$. Then the group $T(P)$ is free abelian generated by the classes of the modules $\Omega_{P}^{1}(k), N_{2}, \ldots, N_{p+1}$, where $N_{i}=\Gamma\left(\left(\Omega_{P}^{-1}(k) \otimes \Omega_{P / S_{i}}^{1}(k)\right)^{\otimes^{2}}\right)$ for $i=2, \ldots, p+1$.

Proof. Let $R$ be the set of all elements of order $p$. We first claim that $R$ is a subgroup and that $R=Q$. Let $u, v \in R$ and $z=v^{-1} u^{-1} v u$. Since $P$ is almost extraspecial, $z$ is central and therefore $(u v)^{p}=u^{p} v^{p} z^{\left(\begin{array}{c}p \\ 2\end{array}\right)}=z^{\left(\begin{array}{c}p \\ 2\end{array}\right)}$. But $z$ has order 1 or $p$ and $p$ divides $\left(\begin{array}{l}p \\ 2\end{array}\right)$ because $p$ is odd. Therefore $u v$ has order $p$ and it follows that $R$ is a subgroup. Now $R$ is a proper subgroup containing the maximal subgroup $Q$, so $R=Q$.

Since every elementary abelian subgroup is contained in $R$, the subgroups $E_{1}, \ldots, E_{p+1}$ are the maximal elementary abelian subgroups of $P$. By Theorem 3.1, the map

$$
\operatorname{Res}: T(P) \longrightarrow \prod_{i=1}^{p+1} T\left(E_{i}\right) \cong \mathbb{Z}^{p+1}
$$

is injective. Since this factors through $T(Q)$, we deduce that $\operatorname{Res}_{Q}^{P}: T(P) \longrightarrow$ $T(Q)$ is injective.

Both (a) and (b) will follow if we prove that the classes $\left[\Omega_{P}^{1}(k)\right],\left[N_{2}\right]$, $\ldots,\left[N_{p+1}\right]$ are mapped under $\operatorname{Res}_{Q}^{P}$ onto the corresponding generators 
of $T(Q)$, namely $\left[\Omega_{Q}^{1}(k)\right],\left[N_{2}^{\prime}\right], \ldots,\left[N_{p+1}^{\prime}\right]$, where $N_{i}^{\prime}=\left(\Omega_{Q}^{-1}(k) \otimes\right.$ $\left.\Omega_{Q / S_{i}}^{1}(k)\right)^{\otimes^{2}}$ for $i=2, \ldots, p+1$. It is clear that $\operatorname{Res}_{Q}^{P}\left(\left[\Omega_{P}^{1}(k)\right]\right)=\left[\Omega_{Q}^{1}(k)\right]$, so we only need to prove that

$$
\operatorname{Res}_{Q}^{P}\left(\left[\Omega_{P / S_{i}}^{1}(k)\right]\right)=\left[\Omega_{Q / S_{i}}^{1}(k)\right]
$$

(these are classes in the Dade groups $D(P)$ and $D(Q)$ respectively). By an easy argument (see also Corollary 4.1.2 in [Bo])

$$
\operatorname{Res}_{Q}^{P}\left(\left[\Omega_{P / S_{i}}^{1}(k)\right]\right)=\left[\Omega_{\operatorname{Res}_{Q}^{P}\left(P / S_{i}\right)}^{1}(k)\right]
$$

and $\operatorname{Res}_{Q}^{P}\left(P / S_{i}\right)$ is a disjoint union of $p$ copies of $Q / S_{i}$, because we can choose central elements in $C_{p^{2}}$ as representatives of $P / Q$. The multiplicity of the $Q$-sets does not change the class of relative syzygies in the Dade group $D(Q)$, by Lemma 3.2.7 in [Bo]. Thus we are left with a single orbit $Q / S_{i}$ and it follows that $\operatorname{Res}_{Q}^{P}\left(\left[\Omega_{P / S_{i}}^{1}(k)\right]\right)=\left[\Omega_{Q / S_{i}}^{1}(k)\right]$.

We now turn to the prime 2. The first extraspecial group to consider is the dihedral group $D_{8}$ of order 8 . But this was done explicitly in [CaTh1]. The group $D_{8}$ has two elementary abelian subgroups $E_{1}, E_{2}$ of rank 2 and the image of the injective map

$$
\alpha: T\left(D_{8}\right) \longrightarrow \mathbb{Z}^{2}
$$

is generated by $\alpha\left(\left[\Omega_{D_{8}}^{1}(k)\right]\right)=(1,1)$ and $\alpha\left(\left[\Omega_{D_{8}}^{-1}(k) \otimes \Omega_{D_{8} / S}^{1}(k)\right]\right)=$ $(0,-2)$, where $S$ is non-central of order 2 . Thus the rational basis in Theorem 3.1 is actually an integral basis of $T\left(D_{8}\right)$.

We do not have to consider the quaternion group of order 8 since it has no elementary abelian subgroup of rank 2 . Actually $T\left(Q_{8}\right)$ has an exceptional behavior described in [CaTh1]. The group $D_{8} * D_{8}$ has maximal elementary abelian subgroups of rank 3 and therefore $T\left(D_{8} * D_{8}\right) \cong \mathbb{Z}$. Thus our next extraspecial group is $D_{8} * Q_{8}$.

Theorem 6.3. Let $p=2$ and let $P=D_{8} * Q_{8}$ be the central product of the dihedral group of order 8 and the quaternion group of order 8 (so $P$ is extraspecial of order 32). Let $E_{1}, \ldots, E_{5}$ be the five maximal elementary abelian subgroups of rank 2, and for each $i$ let $S_{i}$ be a noncentral subgroup of order 2 in $E_{i}$. Then the group $T(P)$ is free abelian generated by the classes of the modules $\Omega_{P}^{1}(k), N_{2}, N_{3}, N_{4}, N_{5}$, where $N_{i}=$ $\Gamma\left(\left(\Omega_{P}^{-1}(k) \otimes \Omega_{P / S_{i}}^{1}(k)\right)^{\otimes^{4}}\right)$ for $2 \leq i \leq 5$.

Proof. The strategy is exactly the same as that of the proof of Theorem 6.1. Let $u, v$ be two elements of order 2 generating $D_{8}$ and let $a, b$ be two generators of $Q_{8}$. Then, up to conjugation, $u, v, u v a, u v b, u v a b$ are the five non-central elements of order 2 and they generate subgroups $S_{1}, \ldots, S_{5}$ respectively. Moreover, together with the central element $a^{2}=b^{2}$, every $S_{i}$ generates one of the five maximal elementary abelian subgroups $E_{i}$ of 
rank 2. We have $C_{P}\left(S_{i}\right) \cong S_{i} \times Q_{8}$ so we need 4-th tensor powers in the description of the generators of $\mathbb{Q} \otimes T(P)$ given by Theorem 3.1. More precisely, Theorem 3.1 asserts that the map $\alpha: T(P) \longrightarrow \mathbb{Z}^{5}$ is injective and that $\alpha\left(\left[\Omega_{P}^{1}(k)\right]\right)=(1,1,1,1,1)$ and $\alpha\left(\left[N_{i}\right]\right)=-8 \cdot e_{i}$, where $e_{i}$ denotes the $i$-th element of the canonical basis of $\mathbb{Z}^{5}$ (with $2 \leq i \leq 5$ ). We have to prove that any class of endo-trivial modules is a linear combination of these.

We follow closely the proof of Theorem 6.1 and indicate the necessary changes. Let $M$ be any endo-trivial $k P$-module. By subtracting a multiple of $\left[\Omega_{P}^{1}(k)\right]$, we can assume that

$$
\alpha([M])=\left(0, s_{2}, s_{3}, s_{4}, s_{5}\right)
$$

and we have to prove that $s_{i}$ is a multiple of 8 for every $i=2, \ldots, 5$. We can assume that $M$ is indecomposable and we proceed by induction on the number of non-zero components in $\alpha([M])$.

Suppose first that $s_{q}$ is the unique non-zero component of $\alpha([M])$, where $q$ is some fixed integer with $2 \leq q \leq 5$, and write $s=s_{q}$ for simplicity. By subtracting a multiple of $\left[N_{q}\right]$, we can assume that $0<s \leq 8$, and we now have to prove that $s=8$. We also know that $s$ is even. The automorphism group of $P$ acts transitively on the five maximal elementary abelian subgroups of $P$, so if $\beta_{i}$ is an automorphism of $P$ mapping $E_{q}$ to $E_{i}$, then the conjugate module $L_{i}=M^{\beta_{i}}$ satisfies

$$
\alpha_{j}\left(\left[L_{i}\right]\right)= \begin{cases}s & \text { if } j=i, \\ 0 & \text { otherwise }\end{cases}
$$

By repeated applications of Theorem 5.1, we obtain

$$
L_{1} \otimes L_{2} \otimes L_{3} \otimes L_{4} \otimes L_{5}=U \oplus \text { (free) }
$$

where $U$ is an indecomposable endo-trivial module such that

$$
\bar{U}=\bar{L}_{1} \oplus \bar{L}_{2} \oplus \bar{L}_{3} \oplus \bar{L}_{4} \oplus \bar{L}_{5} .
$$

We obtain $[U]=\left[\Omega_{P}^{s}(k)\right]$ by injectivity of $\alpha$, hence $U \cong \Omega_{P}^{s}(k)$ because both modules are indecomposable. For some $m$, we have that $\operatorname{Dim}(\bar{M})=2 m$, hence $\operatorname{Dim}(\bar{U})=10 m$ and $\operatorname{Dim}\left(\Omega_{P}^{s}(k)\right)=\operatorname{Dim}(U)=20 m+1$.

Calculations using the MAGMA computer algebra system [BoCa] yield

$$
\begin{aligned}
& \operatorname{Dim}\left(\Omega_{P}^{2}(k)\right)=3 \cdot 32+1, \\
& \operatorname{Dim}\left(\Omega_{P}^{4}(k)\right)=9 \cdot 32+1, \\
& \operatorname{Dim}\left(\Omega_{P}^{6}(k)\right)=14 \cdot 32+1, \\
& \operatorname{Dim}\left(\Omega_{P}^{8}(k)\right)=15 \cdot 32+1
\end{aligned}
$$

(actually the mod-2 cohomology of $P$ can be computed using Quillen's theorem $[\mathrm{BeCa}]$ and the dimensions of the cohomology groups turn out to 
be $1,4,9,15,21,26,29,30, \ldots)$. We know that $\operatorname{Dim}\left(\Omega_{P}^{s}(k)\right)=20 m+1$ but the only value of $s$ for which $5 \operatorname{divides} \operatorname{Dim}\left(\Omega_{P}^{s}(k)\right)-1$ is $s=8$. This proves the first step of induction.

For the second part of the proof, we have to assume that the number of non-zero components in $\alpha([M])$ is at least 2 . An important difference in the argument concerns the periodicity of $\bar{M}$. We have $S_{1}<E_{1}<S_{1} \times Q_{8}$ and we know that $\left[M \downarrow_{E_{1}}^{P}\right]=0$. But

$$
\operatorname{Res}_{E_{1}}^{S_{1} \times Q_{8}}: T\left(S_{1} \times Q_{8}\right) \longrightarrow T\left(E_{1}\right)
$$

is an isomorphism because $E_{1}$ is the only maximal elementary abelian subgroup of $S_{1} \times Q_{8}$ (Theorem 3.1). Therefore $\left[M \downarrow_{S_{1} \times Q_{8}}^{P}\right]=0$, that is,

$$
M \downarrow \stackrel{P}{P}{ }_{S_{1} \times Q_{8}} \cong k \oplus \text { (free) } .
$$

By Lemma 4.1, it follows that $\bar{M}$ is free on restriction to $\overline{S_{1} \times Q_{8}}$. Since $S_{1} \times Q_{8}$ is a maximal subgroup of $P$ we deduce that $\bar{M}$ is a periodic $k \bar{P}$-module (Lemma 5.3 in [CaTh2]).

The rest of the proof is exactly the same as that of Theorem 6.1. We apply Theorem 4.3 to construct a module $L$ such that $\alpha([L])$ has a unique non-zero component and we apply induction to $[L]$ and $[M]-[L]$.

Finally we consider almost extraspecial groups for the prime 2 . The only case to treat is the following group of order 16.

Theorem 6.4. Let $p=2$ and let $P=D_{8} * C_{4}$ be the central product of the dihedral group of order 8 and the cyclic group of order 4 (so $P$ is almost extraspecial of order 16). Let $E_{1}, E_{2}, E_{3}$ be the three maximal elementary abelian subgroups of rank 2 , and for each $i$ let $S_{i}$ be a non-central subgroup of order 2 in $E_{i}$. Then the group $T(P)$ is free abelian generated by the classes of the modules $\Omega_{P}^{1}(k), N_{2}, N_{3}$, where $N_{i}=\Gamma\left(\left(\Omega_{P}^{-1}(k) \otimes \Omega_{P / S_{i}}^{1}(k)\right)^{\otimes^{2}}\right)$ for $2 \leq i \leq 3$.

Proof. The strategy is exactly the same as that of the proof of Theorem 6.3 and we only indicate the changes. Let $u, v$ be two elements of order 2 generating $D_{8}$ and let $a$ be a generator of $C_{4}$. Then, up to conjugation, $u, v, u v a$ are the three non-central elements of order 2 and they generate subgroups $S_{1}, S_{2}, S_{3}$ respectively. Moreover, together with the central element $a^{2}$, every $S_{i}$ generates one of the three maximal elementary abelian subgroups $E_{i}$ of rank 2. We have $C_{P}\left(S_{i}\right) \cong S_{i} \times C_{4}$ so we need second tensor powers in the description of the generators of $\mathbb{Q} \otimes T(P)$ given by Theorem 3.1.

More precisely, Theorem 3.1 asserts that the map $\alpha: T(P) \longrightarrow \mathbb{Z}^{3}$ is injective and that $\alpha\left(\left[\Omega_{P}^{1}(k)\right]\right)=(1,1,1), \alpha\left(\left[N_{2}\right]\right)=(0,-4,0)$, and $\alpha\left(\left[N_{3}\right]\right)=(0,0,-4)$. We have to prove that any class of endo-trivial modules is a linear combination of these elements. 
In the first case where $\alpha([M])$ has a unique non-zero component $s$, we can assume that $s$ is even and $s \leq 4$ and we have to prove that $s=4$. We construct again an indecomposable endo-trivial module $U$ such that

$$
\bar{U}=\bar{L}_{1} \oplus \bar{L}_{2} \oplus \bar{L}_{3},
$$

where $L_{i}$ is a conjugate of $M$ by an automorphism of $P$. We then obtain $U \cong \Omega_{P}^{s}(k)$. We have $\operatorname{Dim}(\bar{M})=2 m$ for some $m$, hence $\operatorname{Dim}(\bar{U})=6 m$ and $\operatorname{Dim}\left(\Omega_{P}^{s}(k)\right)=\operatorname{Dim}(U)=12 m+1$. On the other hand, by direct computation (e.g. using the MAGMA computer algebra system) we obtain $\operatorname{Dim}\left(\Omega_{P}^{2}(k)\right)=33$ and $\operatorname{Dim}\left(\Omega_{P}^{4}(k)\right)=49$. Thus the only possibility is $s=4$.

In the second part of the proof, we assume that the number of non-zero components in $\alpha([M])$ is at least 2 . We have again $\left[M \downarrow_{E_{1}}^{P}\right]=0$ and we need to prove that $M$ is periodic. But we have $S_{1}<E_{1}<S_{1} \times C_{4}$ and

$$
\operatorname{Res}_{E_{1}}^{S_{1} \times C_{4}}: T\left(S_{1} \times C_{4}\right) \longrightarrow T\left(E_{1}\right)
$$

is an isomorphism because $E_{1}$ is the only maximal elementary abelian subgroup of $S_{1} \times C_{4}$. Therefore $\left[M \downarrow_{S_{1} \times C_{4}}^{P}\right]=0$, that is,

$$
M \downarrow_{S_{1} \times C_{4}}^{P} \cong k \oplus \text { (free) } .
$$

By Lemma 4.1, it follows that $\bar{M}$ is free on restriction to $\overline{S_{1} \times C_{4}}$. Since $S_{1} \times C_{4}$ is a maximal subgroup of $P$ we deduce that $\bar{M}$ is a periodic $k \bar{P}$ module. The rest of the proof is exactly the same as that of Theorem 6.3.

\section{The general case}

The following is the main theorem of the paper. It, together with the results of [CaTh2], give a complete classification of endo-trivial modules for $p$ groups.

Theorem 7.1. Let $P$ be a finite p-group having a maximal elementary abelian subgroup of rank 2 and suppose that $P$ is not semi-dihedral. Fix the same notation as in Theorem 3.1. Then the group $T(P)$ is free abelian generated by the classes of the modules $\Omega_{P}^{1}(k), N_{2}, \ldots, N_{r}$.

Proof. Consider the injective map $\alpha: T(P) \longrightarrow \mathbb{Z}^{r}$ of Theorem 3.1. We know that $\alpha\left(\left[\Omega_{P}^{1}(k)\right]\right)=(1,1, \ldots, 1)$ and that

$$
\alpha\left(\left[N_{i}\right]\right)=-n_{i} p \cdot e_{i} .
$$

Here, $e_{i}=(0, \ldots, 0,1,0, \ldots, 0)$ the $i$-th element of the canonical basis of $\mathbb{Z}^{r}$. We set $n_{i}=1$ (resp. 2, resp. 4) if $C_{P}\left(S_{i}\right) / S_{i} \cong L_{i}$ is cyclic of order 2 (resp. cyclic of order $\geq 3$, resp. generalized quaternion). We have 
to prove that any class of endo-trivial modules is a linear combination of these elements.

Let $M$ be any endo-trivial $k P$-module. By subtracting a multiple of $\left[\Omega_{P}^{1}(k)\right]$, we can assume that $\alpha_{1}([M])=0$ and we then have to prove that $[M]$ is a linear combination of $\left[N_{2}\right], \ldots,\left[N_{r}\right]$. In other words

$$
\alpha([M])=\left(0, s_{2}, s_{3}, \ldots, s_{r}\right)
$$

and we have to prove that $s_{i}$ is a multiple of $n_{i} p$ for every $i=2, \ldots, r$. Without loss of generality, we can take $i=2$.

By Lemma 2.2, we know that $N_{P}\left(E_{2}\right)=R * L$, where $R=E \cdot E_{2}$ is extraspecial of order $p^{3}$ and $L=L_{2}$ is either cyclic or generalized quaternion. Recall that $R$ is extraspecial of exponent $p$ if $p$ is odd and that $R=D_{8}$ if $p=2$. Clearly $L$ contains a subgroup $L^{\prime}$ defined as follows:

$$
L^{\prime}= \begin{cases}C_{p} & \text { if } p \text { is odd }, \\ C_{2} & \text { if } p=2 \text { and } L=C_{2}, \\ C_{4} & \text { if } p=2 \text { and } L=C_{2^{m}}, m \geq 2, \\ Q_{8} & \text { if } p=2 \text { and } L=Q_{2^{m}}, m \geq 3 .\end{cases}
$$

Then $N_{P}\left(E_{2}\right)$ contains a subgroup $Q=R * L^{\prime}$ which is of the following type:

$$
Q= \begin{cases}R & \text { if } p \text { is odd } \\ D_{8} & \text { if } p=2 \text { and } L=C_{2}, \\ D_{8} * C_{4} & \text { if } p=2 \text { and } L=C_{2^{m}}, m \geq 2, \\ D_{8} * Q_{8} & \text { if } p=2 \text { and } L=Q_{2^{m}}, m \geq 3 .\end{cases}
$$

The restriction map $T(P) \rightarrow T\left(E_{2}\right)$ factors through $T(Q)$ and so does the map $\alpha$. Thus we need only consider $\left[M \downarrow_{Q}^{P}\right] \in T(Q)$. The map of Theorem 3.1 for the group $Q$ is an injection $\alpha_{Q}: T(Q) \longrightarrow \mathbb{Z}^{r^{\prime}}$ (for a certain $\left.r^{\prime}\right)$ and the first two components of $\alpha_{Q}\left(\left[M \downarrow_{Q}^{P}\right]\right)$ are the same as those of $\alpha([M])$, that is, 0 and $s_{2}$. We now conclude that $s_{2}$ is a multiple of $n_{2} p$ by applying the results of Sect. 6 for the group $Q$ in each of the appropriate cases. More precisely, if $p$ is odd, then $Q=R$ is extraspecial of order $p^{3}$ and exponent $p$, we have $n_{2}=2$ and Theorem 6.1 applies. If $p=2$, then we have to apply Theorem 6.4 if $Q=D_{8} * C_{4}$ (with $n_{2}=2$ ), and apply Theorem 6.3 provided $Q=D_{8} * Q_{8}$ (with $n_{2}=4$ ). We apply the remarks before Theorem 6.3 in the case that $Q=D_{8}$ (with $n_{2}=1$ ).

Corollary 7.2. Let $P$ be a finite p-group. Then $T(P)$ is generated by the classes of relative syzygies of the trivial module, except in the case that $p=2$ and $P$ is generalized quaternion.

Proof. If $P$ is generalized quaternion, it is known that there are exceptional endo-trivial modules (see [CaTh1]), so we exclude this case. The result is well-known (and easy) if $P$ is cyclic and it can be found in Theorem 7.1 
of [CaTh1] if $P$ is semi-dihedral. If now $P$ has a maximal elementary abelian subgroup of rank 2, then the result follows from Theorem 7.1 above. If all maximal elementary abelian subgroups have rank $\geq 3$, then $T(P)$ is generated by $\left[\Omega_{P}^{1}(k)\right]$ (Corollary 1.3 in $\left.[\mathrm{CaTh} 2]\right)$.

\section{References}

[Al] Alperin, J.L.: A construction of endo-permutation modules. J. Group Theory 4, 3-10 (2001)

[BeCa] Benson, D.J., Carlson, J.F.: The cohomology of extraspecial groups. Bull. Lond. Math. Soc. 24, 209-235 (1992)

[BoCa] Bosma, W., Cannon, J.: Handbook of Magma Functions. Sydney: Magma Computer Algebra 1999

[Bo] Bouc, S.: Tensor induction of relative syzygies. J. Reine Angew. Math. 523, 113-171 (2000)

[BoMa] Bouc, S., Mazza, N.: The Dade group of (almost) extraspecial p-groups. J. Pure Appl. Algebra. To appear

[BoTh] Bouc, S., Thévenaz, J.: The group of endo-permutation modules. Invent. Math. 139, 275-349 (2000)

[Ca] Carlson, J.F.: Constructing endotrivial modules. Preprint (2004)

[CaTh1] Carlson, J.F., Thévenaz, J.: Torsion endo-trivial modules. Algebr. Represent. Theory 3, 303-335 (2000)

[CaTh2] Carlson, J.F., Thévenaz, J.: The classification of torsion endo-trivial modules. Ann. Math. To appear

[Da] Dade, E.C.: Endo-permutation modules over p-groups, I, II. Ann. Math. 107, 459-494 (1978); 108, 317-346 (1978)

[Go] Gorenstein, D.: Finite Groups. New York: Harper \& Row 1968

[GLS] Gorenstein, D., Lyons, R., Solomon, R.: The classification of the finite simple groups. Number 2, Math. Surveys and Monographs, Vol. 40.2. Providence: Am. Math. Soc. 1996

[Ma] Mazza, N.: The Dade group of a metacyclic p-group. J. Algebra 266, 102-111 (2003)

[Pu] Puig, L.: Affirmative answer to a question of Feit. J. Algebra 131, 513-526 (1990) 